\title{
Stellar convection with nuclear burning
}

\author{
David Arnett, Casey Meakin \\ and Patrick A. Young \\ Steward Observatory, University of Arizona, Tucson AZ 85721, USA \\ email: darnett@as.arizona.edu
}

\begin{abstract}
Careful choice of of method, problem, and zoning has allowed us to do threedimensional (3D) simulations of thermally relaxed, nearly adiabatic convection (with nuclear burning). The simulations are run long enough so that a robust statistical state is found. We find that 2D simulations are biased relative to 3D simulations: 2D shows larger velocities and less mixing than their 3D counterparts. Detailed theoretical analysis of these numerical experiments allows us to begin to build a simple theoretical model of turbulent convection in stars, which may be used in 1D calculations of stellar evolution. Implications for stellar evolution, will be discussed. Oxygen shell burning simulations in 3D, and multishell burning of $\mathrm{C}, \mathrm{Ne}, \mathrm{O}$, and $\mathrm{Si}$ in 2D will be presented, as will aspherical distortion in supernovae progenitors (Meakin and Arnett, 2006a). Contact will be made with convective driving of waves, convective zone growth by entrainment, the velocity scale and the geometric parameters in mixing length theory, and the solar Ne abundance problem. Explicit comparisons of compressible and anelastic methods at modest Mach numbers ( $\mathcal{M} \approx 0.01$ to 0.1 ), as well as solutions of the nonradial wave equations, are presented here. Additional detail is presented in the poster by Meakin.
\end{abstract}

Keywords. Convection, nucleosynthesis, neutrinos, turbulence, waves, methods: numerical, Sun: helioseismology, stars: evolution, stars: supernovae

\section{3D simulations with modest resources.}

Three dimensional, time dependent simulations, with good resolution, are expensive in terms of computer usage. Obtaining meaningful results with modest computing resources requires many careful choices. Some of ours are:

- Oxygen burning allows thermal relaxation for quasi-adiabatic convection. Neutrino cooling and nuclear heating times at the bottom of the oxygen burning shell are closer to the sound travel time than is the cooling time by radiative diffusion at the bottom of the solar convective zone to the corresponding sound travel time. Thus, relaxation to thermal equilibrium takes fewer time steps for oxygen burning.

- The computational domain is a subsection of the star, to lower the computational load. This means we must ignore lower order modes and rotation which are not contained by these small domains. Since rotation must be ignored we also ignore MHD effects, which helioseismology leads us to suspect is coupled to rotation. See Figure 1 for a visual representation of some of our domains.

- Use of multi-fluid, realistic physics (nuclear reactions, neutrino emission, equation of state) allows us to use realistic initial models and make astronomical connections.

- Careful treatment of boundary conditions and mapping of 1D initial models into 3D allows us to begin with low noise models and to simulate for long times. We use an inner reflecting boundary, an outer stress-free boundary, and periodic boundaries on the sides (2 or 4$)$. 


\section{Computational Domains}
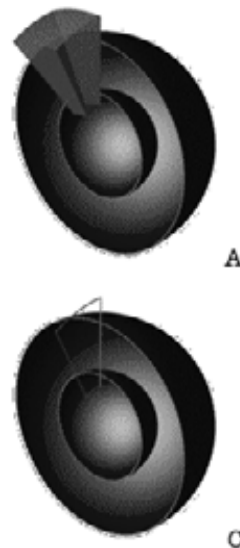

A.

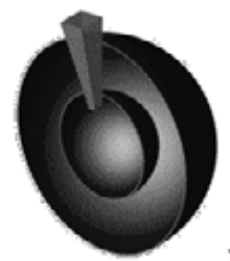

B.

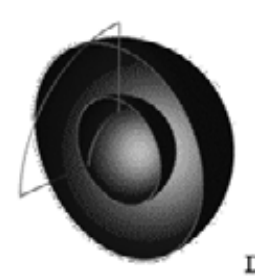

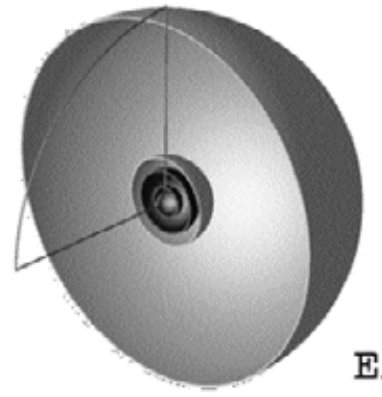

$\mathrm{E}$

Figure 1. Computational Domains. The hemispheres represent the radial extent of compositional interfaces, while the sectors and wedges represent the computational domains for our $2 \mathrm{D}$ and $3 \mathrm{D}$ simulations.

- Extensive graphical and analysical tools were used to understand and evaluated the competing physical processes, and derive a significant simplification of complex, dynamic phenomena.

\section{Dynamic interactions in stratified media.}

Stably stratified fluids are fundamentally different dynamical entities from convectively unstable fluids. Stable stratification means that there is a restoring force to maintain the stratification, while convectively unstable fluids have growth of perturbations. A feature of mixing-length theory (as used in stellar physics) is that gradients at the boundaries of convective regions are ignored; thus the theory cannot properly deal with such boundaries. However, stars evolve by developing interfaces in composition; this is most noticable at the boundaries of convective zones. Composition changes are due to thermonuclear burning, or gravitational settling.

Figure 2 shows the dynamics of a fluid interface, which is common terrestrially: think of surface waves on a lake. The convective plumes rise until they encounter the stable interface. They distort the interface, changing kinetic energy into potential energy of the distortion of the stable region just above the interface. This distortion is releaved by a release of stress and wave generation. This is the process which is not included in mixing length theory, and the one which explains how c onvection stops at the boundary. Note that acceleration has the opposite sign from velocity as the plume stops, as it should. The conditions which determine the boundary between convective and nonconvective regions are (1) dynamic, not static, and (2) nonlocal, not local, as the motion of plumes and of waves implies.

\section{3. $2 \mathrm{D}$ versus $3 \mathrm{D}$ simulations.}

Why do we consider 2D simulations at all, if we have a 3D phenomena? It is about one hundred times cheaper to do a 2D simulation than the corresponding $3 \mathrm{D}$ one, with typical resolution in use today. Since budgets are not logarithmic, this explains the attraction 


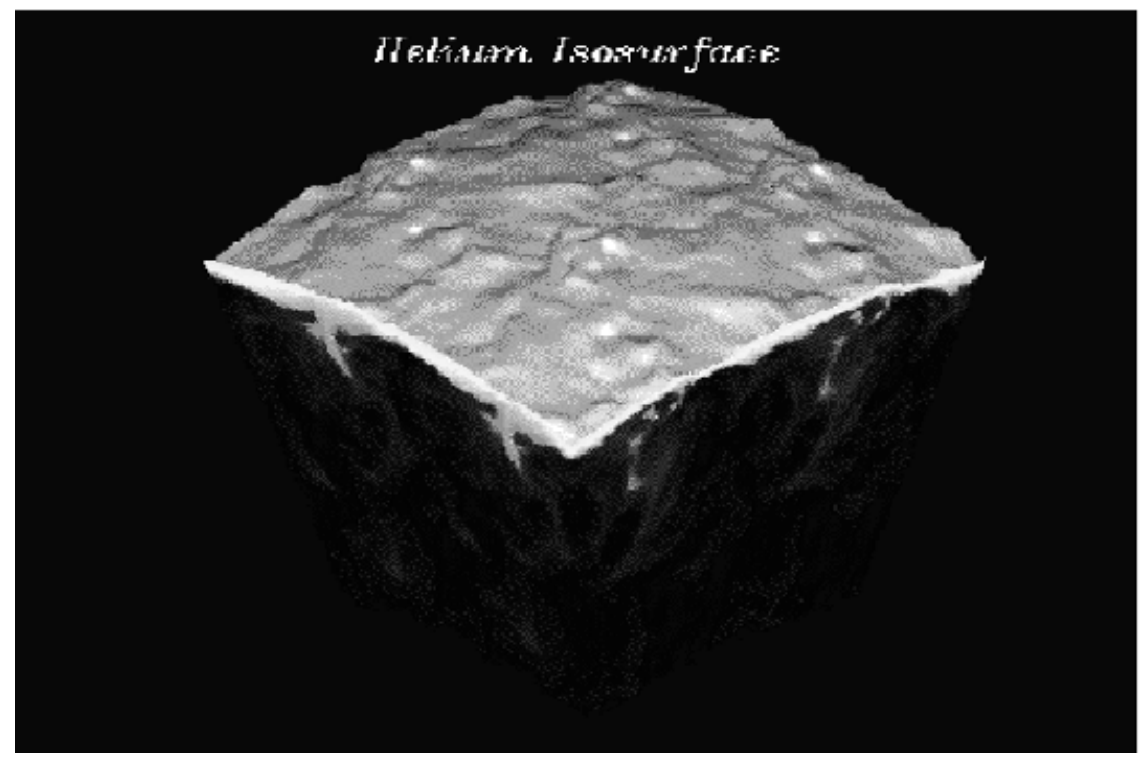

Figure 2. Distortion of Convective Interfaces. The light colored surface represents an interface between a hydrogen rich region (above) and a hydrogen depleted (i.e., helium rich) region (below), which is convective. Convection drives wavelike distortions in the surface, which cause waves to radiate above it. Because the convective motion is slow, it couples better to the slower g-modes than the faster p-modes.
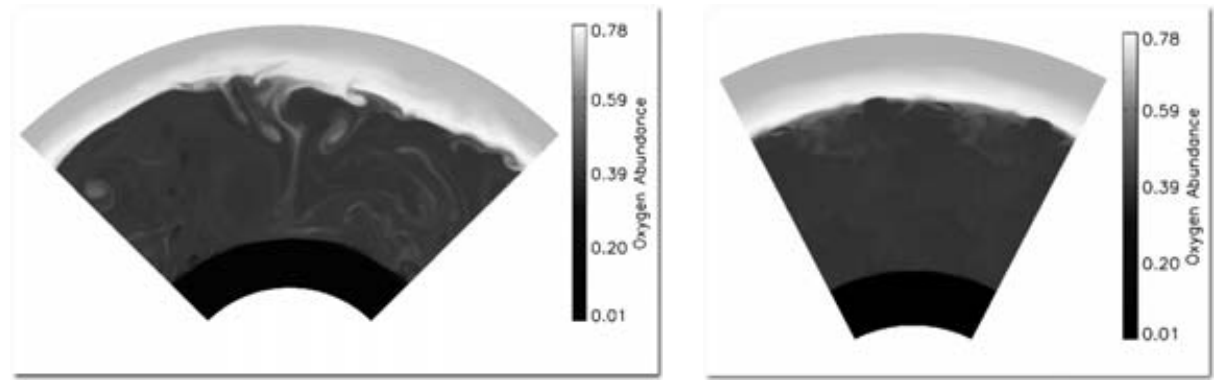

Figure 3. Oxygen abundance in a 2D (left) and 3D (right) simulation of the oxygen burning shell, using the same colormap. The cyclonic patterns are more pronounced in $2 \mathrm{D}$, and the composition contrasts are larger and last longer. See Meakin \& Arnett (2006c).

of using $2 \mathrm{D}$ as a shortcut to 3D. We have directly compared $2 \mathrm{D}$ and $3 \mathrm{D}$ simulations for stellar convection and find that 2D is highly biased and will be misleading if not used with caution. In particular, in $2 \mathrm{D}$ the velocity scale for vortices is as much as a factor of 8 larger than 3D. At the same time, the mixing efficiency is much lower in $2 \mathrm{D}$. This is because $3 \mathrm{D}$ vortices are unstable, while in $2 \mathrm{D}$ they are pinned to the $2 \mathrm{D}$ surface. One characteristic of $2 \mathrm{D}$ is easily visible; large cyclonic patterns develop and do not readily decay. These correspond to hurricanes and typhoons on earth, which are really almost $2 \mathrm{D}$ because the height of the atmosphere is much less than their diameter.

This may be seen by comparing Fig. 3, which shows the $2 \mathrm{D}$ the $3 \mathrm{D}$ simulations of oxygen burning. The oxygen abundance is shown. Notice the larger contrast in $2 \mathrm{D}$ relative to $3 \mathrm{D}$.

Having warned the reader, we now proceed to make use of the computational advantage of 2D: we examine a computational volume large enough to contain multiple nuclear 


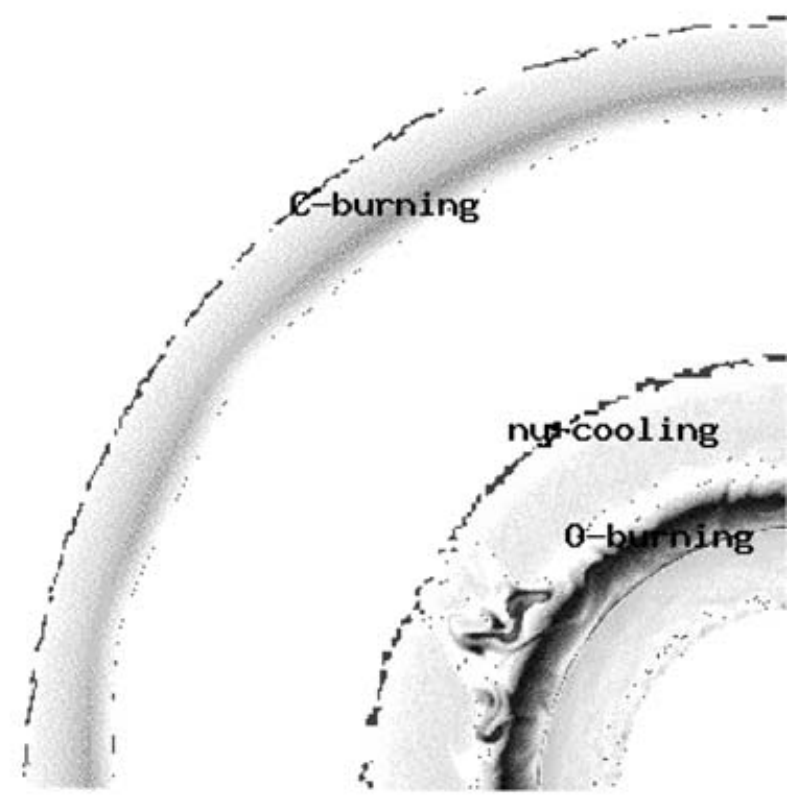

Figure 4. Multiple Burning Shells (C,Ne,O,Si). Nuclear heating and neutrino cooling are shown by a colormap; the radial structure for a quadrant is shown. The outer shaded band is the C-burning shell. The darkest region is the O-burning shell; it lies below a neutrino-cooled region, and is convectively coupled to it. See Meakin \& Arnett (2006a).

burning shells. The star is a 23 solar mass presupernova progenitor, undergoing shell burning of $\mathrm{H}, \mathrm{He}, \mathrm{C}, \mathrm{Ne}, \mathrm{O}$, and Si just prior to core collapse. We expect that the two brightest stars in Orion, Betelgeuse and Rigel, may develop an analagous internal structure. Only for $\mathrm{H}$ and He burning is the heating from nuclear burning countered by radiative cooling by photon diffusion. For the later burning stages, direct emission of neutrino-antineutrino pairs compensates for nuclear heating. This vigorous cooling means that thermal relaxation occurs on a timescale nearer to the burning timescale, so this exotic stage is easier to simulate than the bottom of the convection zone of the Sun.

The convective coupling of a neutrino cooling region with an underlying nuclear heating one was predicted (see Arnett (1996) for details). Figure 4 shows two unanticipated phenomena (which are spectacular in the movie, but more subtle in the still): (1) ingestion of new fuel into the flame zone for oxygen burning, and (2) distortion of the C-burning shell away from spherical symmetry due to interaction with the oxygen burning shell (mediated by wave motion). The 2D simulations overemphasize the velocity scales, but these phenomena will occur. Because the burning becomes more vigorous as the core approaches collapse, and the restoring forces become weaker, it is likely that the collapsing core will have large deviations from the spherical geometry traditionally assumed by builders of presupernova models. This is without any significant rotation, which will also break the spherical symmetry.

\section{4. $3 \mathrm{D}$ results}

\subsection{Density variations at convective-nonconvective boundaries}

A convenient way to characterize a stable region adjacent to an convectively unstable one is the buoyancy frequency (or the Brunt-Väisälä frequency), $N^{2}=\left(\nabla_{a}+\nabla_{x}-\nabla\right) g / H_{P}$ 


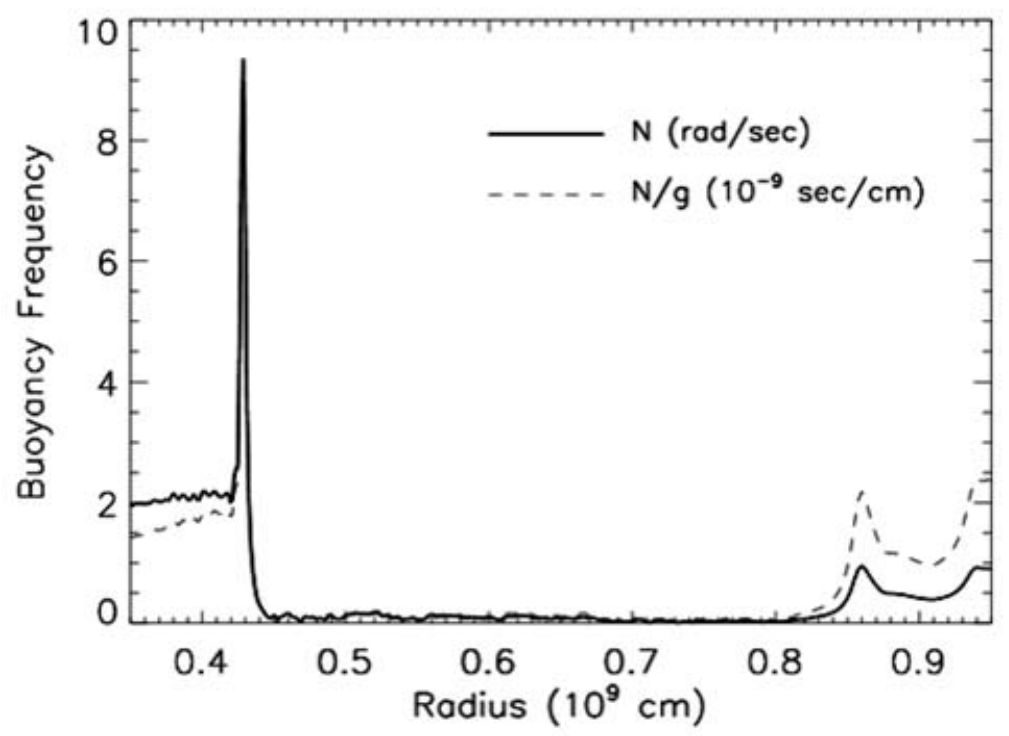

Figure 5. Buoyancy Frequency versus Radius. Spikes appear at the edge of the convective region; here the fluid becomes more resistant to radial displacement. See Meakin \& Arnett (2006a)

(see Kippenhahn \& Weigert (1990), p.41). This is shown in Figure 5 as a function of radius; the magnitude of $N$ is plotted, but note that $N^{2}$ is negative in an unstable layer. The convective zone lies in the range $r=0.44 \times 10^{9} \mathrm{~cm}$ to $0.84 \times 10^{9} \mathrm{~cm}$; here $N^{2}<0$ so the frequencies are imaginary, a sign that exponentiating solutions are possible (in this case turbulent convection).

Figure 6 shows that the angle averaged r.m.s. deviation of density $\delta \rho / \rho$ has a similar behavior versus radius. A spike in the buoyancy frequency near $r=0.44 \times 10^{9} \mathrm{~cm}$ corresponds to a spike in $\delta \rho / \rho$. This is the region in which the convective plumes are distorting the stable layers. Because the buoyancy frequency is high, the plumes are quickly stopped, and the distortion of the stable matter gives a change in density. The change is not a compression, but a change in the geometry of the interface. Imagine a lake with waves. The average level of the water corresponds to the radius $r$. The instantaneous level of the water changes, as air and then water cross that radius. The change is seen to be large in a fixed (eulerian) coordinate system, but is seen to be smaller from a comoving (lagrangian) system. There is significant energy in these waves; their luminosity (directed away from the convective region) is balanced by the energy flux in the convective plumes (directed primarily toward the interface).

\subsection{Connection with linear analysis: waveforms}

An important theoretical result is that we can clearly identify motions in the convectively stable regions with linear waves (Unno,Osaki,Ando, Saio, \& Shibahashi (1989)); this is shown in Fig. 7. Although nonlinear calculations are necessary to determine the amplitudes, the wave forms are well represented by the linear solutions. In addition to confirming that our numerical methods give accurate waveforms, it will also allow a new tool to be applied to this problem. 


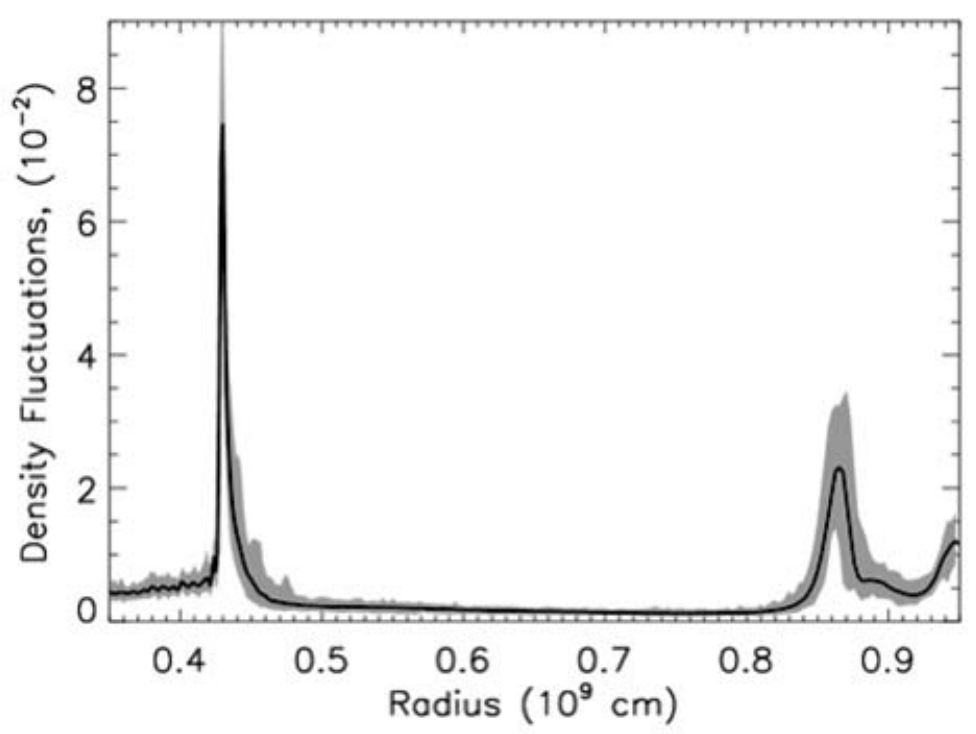

Figure 6. Density Variations versus Radius. The solid line shows the r.m.s. density fluctuation; it has peaks at the top and bottom of the convection region. The larger peak at the bottom is due to the larger stiffness there (see Figure 4 and Meakin \& Arnett (2006a), Meakin \& Arnett (2006b)). The shaded region indicates the range of fluctuation.

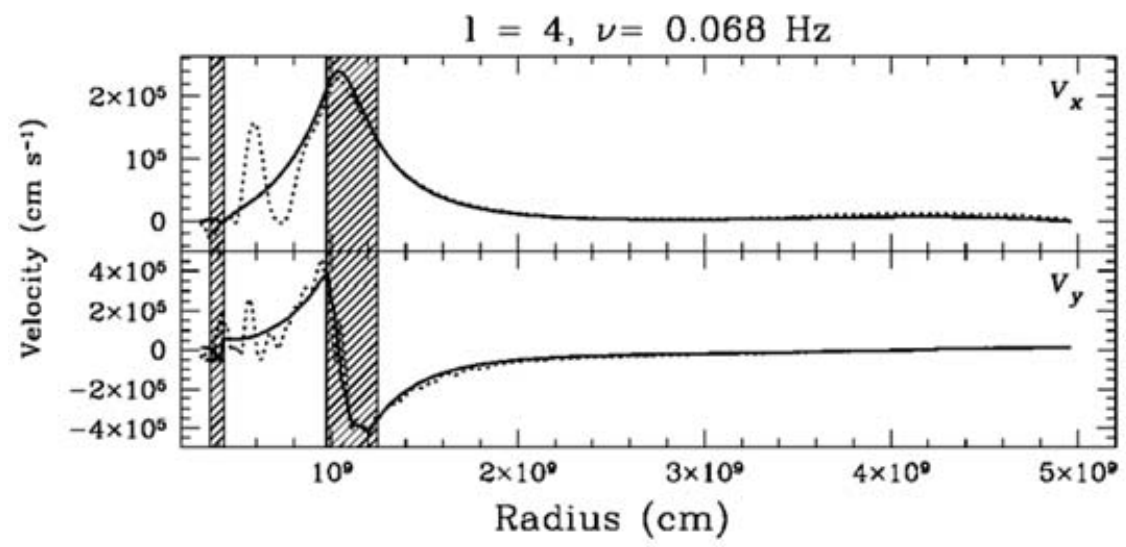

Figure 7. Comparison of numerical and analytic waveforms. The convective region lies between the diagonally-shaded vertical bars. The numerical waveforms are shown as dotted lines; the linear waves, scaled in amplitude, are shown as the solid curves. Except in the convective region (where they should not agree) the two agree remarkably well. (see Meakin \& Arnett (2006a), Meakin \& Arnett (2006b)).

\subsection{The Mixing-Length parameter}

Mixing-length theory has been used in stellar evolution for almost half a century, so it must be doing some thing right. We can now investigate what is right and wrong. Fig. 8 compares the functional forms for convective speed squared, and the convective thermal flux, in terms of the superadiabatic temperature gradient. Within the convection zone the agreement is better than I would have expected (2D results give worse agreement). 


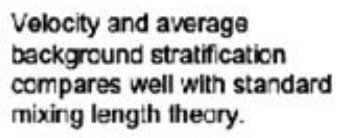

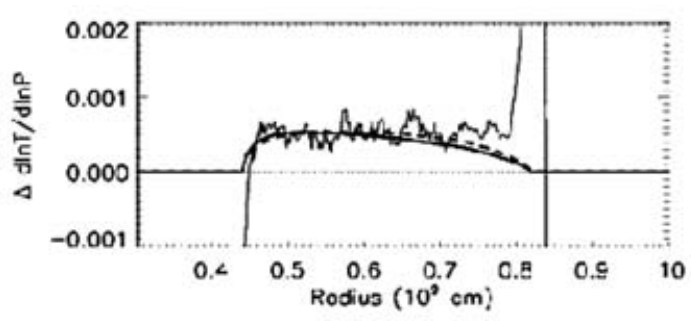

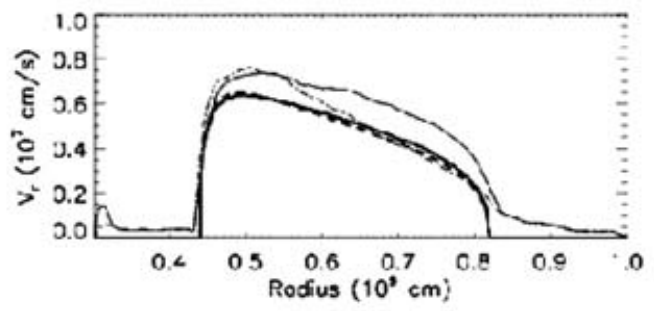

Figure 8. Turbulent Compressible Convection: Comparison with Mixing-Length Theory. Mixing-length theory is shown as dark solid lines for (top) the superadiabatic temperaure excess and (bottom) convective speed. (see Meakin \& Arnett (2006a), Meakin \& Arnett (2006b)).

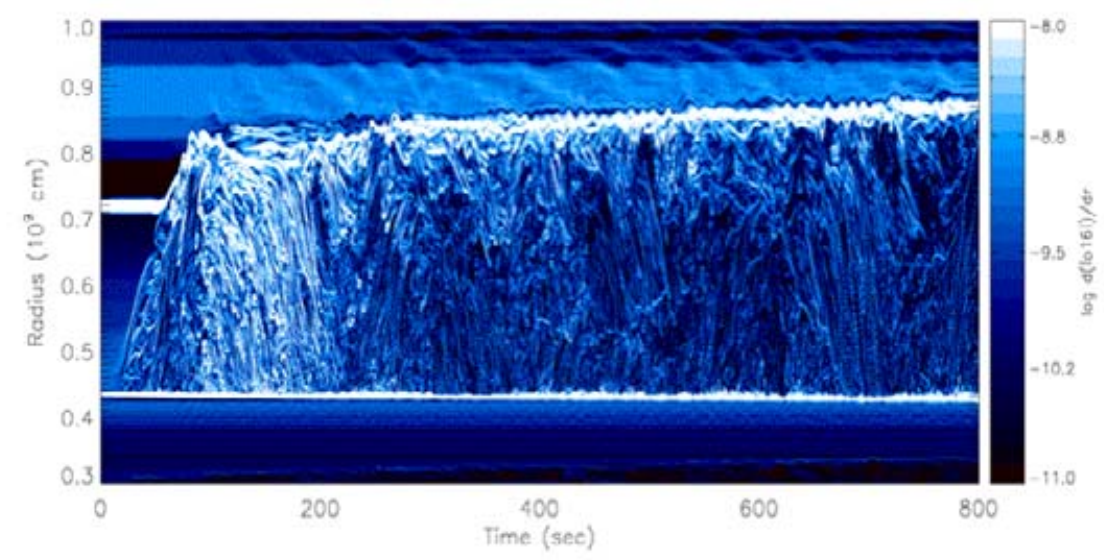

Figure 9. Mixing as a function of radius (y-axis) and time (x-axis). The variable shown is a measure of the abundance gradient (white is large, black is zero). (see Meakin \& Arnett (2006a), Meakin \& Arnett (2006b)).

Outside the convection zone there is wave motion and wave energy flux which are not captured by mixing-length theory.

\subsection{New physics: turbulent entrainment in stars}

Fig. 9 shows the evolution of mixing in the oxygen burning shell. The variable shown is a measure of the abundance gradient (white is large, black is small), and traces the mixing in space and time. The results are reassuringly robust in a statistical sense. We see that within about 100 seconds the convective region settles down into a quasi-stationary state; this is the thermal relaxation time. However, there is a secular growth in the size of the convective region. This is related to the distortion of the boundaries with stable regions 


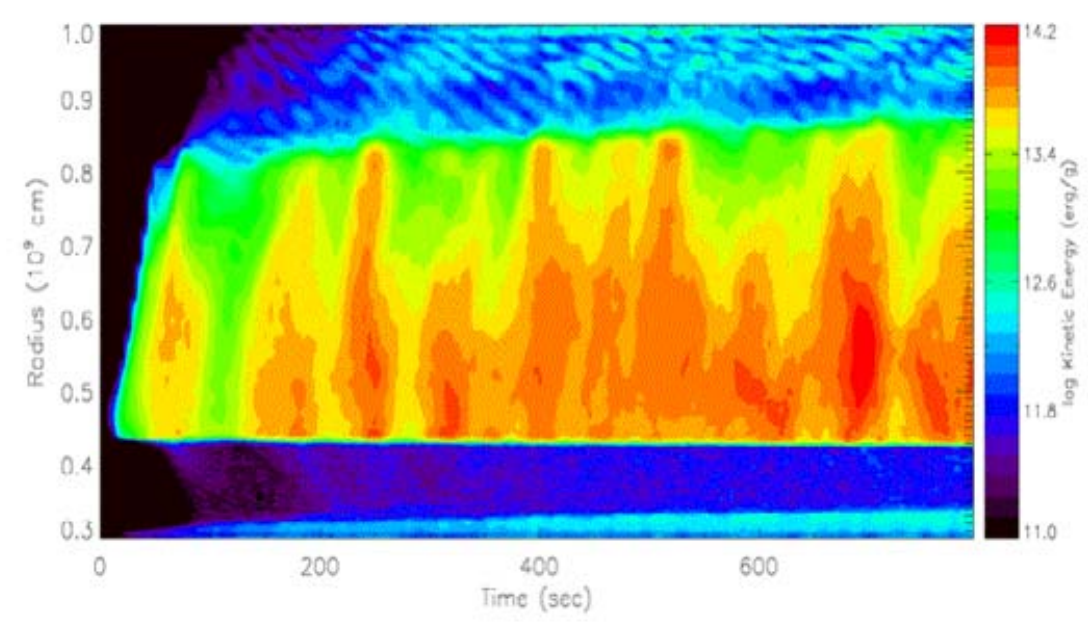

Figure 10. Mixing as a function of radius (y-axis) and time (x-axis). The variable shown is the convective kinetic energy. The fluctuation in time reflect turbulent intermittency. (see Meakin \& Arnett (2006a), Meakin \& Arnett (2006b)).

by plumes and the radiation of waves. The growth is faster at the outer boundary, which is less stiff than the inner (lower buoyancy frequency, see Fig. 5). However, the lower boundary does move inward, although at a slower rate. Fig. 10 shows the evolution of turbulent kinetic energy in the oxygen burning shell. A strong intermittency is evident, but the statistical properties are robust. Wave radiation (primarily g-modes) is evident at the top of the convective zone.

\subsection{Implications for the Standard Solar Model}

We have a detailed simulation only for one specific case, and do not know how universal (if at all) such behavior is. The robustness and physical plausibility tempt us to generalize these 3D numerical results into a simplified physical model of 3D convection, which may be used to replace mixing length theory in stellar evolutionary calculations. We begin by considering the Sun and the "solar neon problem" (see Asplund, this Symposium).

The problem is that helioseismologically measured sound speeds are not simultaneously compatible with (1) the standard solar model, and (2) the new solar abundances, inferred with use of 3D hydrodynamic solar atmospheres. In particular, the theoretical convective zone does not reach deeply enough into the sun, and the sound speeds show a strong deviation from the experimental ones below the bottom of the convective zone. Bahcall suggested that this could be fixed by an additional source of opacity below the convection zone. As the opacity calculations are consistent between the Opacity Project group and the OPAL group at LLNL, it was suggested the the poorly know Ne abundance was much larger, to provide the extra opacity.

Fig. 11 show the superadiabatic gradient in a solar model. It is strongly positive only in a narrow layer near the photosphere. In this region, radiative transfer, not readiative diffusion, is required (see R. Stein, this Symposium). However, for almost all of the convection zone, either by volume or by mass, the fluid motion is almost adiabatic, like our simulation.

Using our result that waves are generated at the lower boundary of the solar convection zone, we examine the radiative damping of these waves as they propagate inward; 


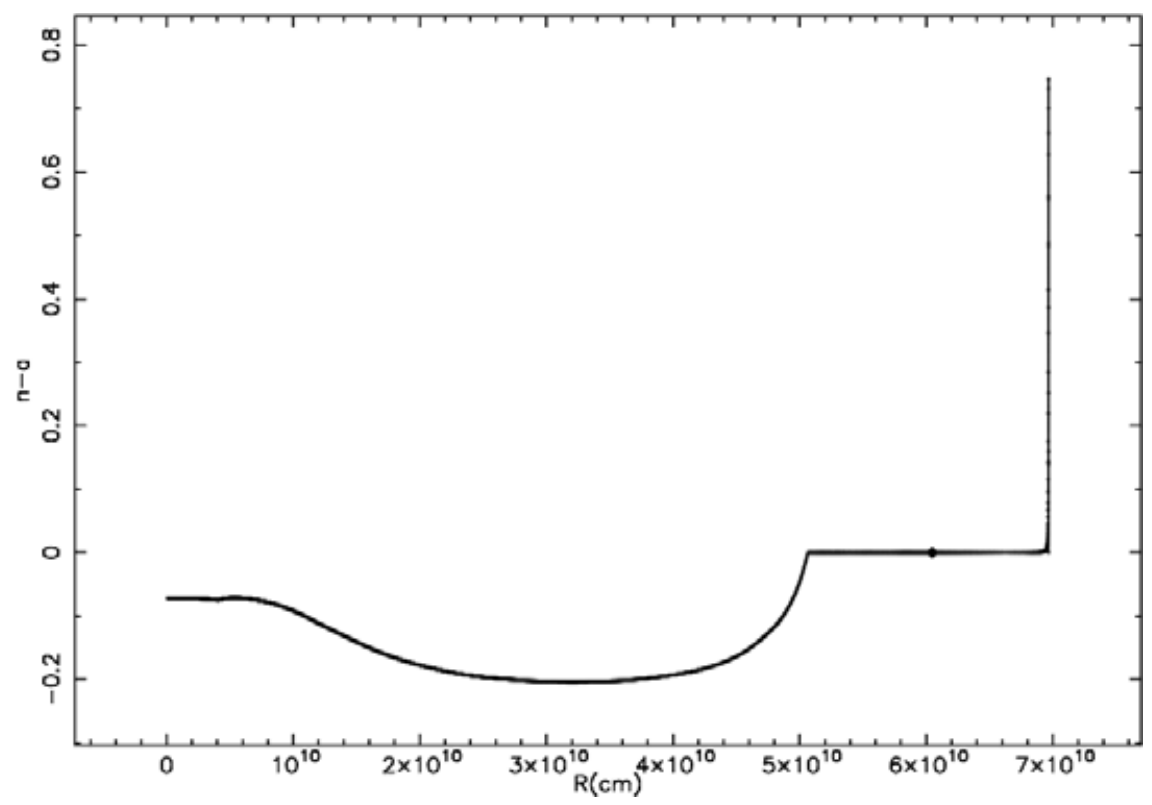

Figure 11. The superadiabatic gradient, $\nabla-\nabla_{a}-\nabla_{x}$, versus radius, for a solar model. The gradient is superadiabatic (strongly positive) only in a narrow layer just below the photosphere. Most solar (and stellar) convection is nearly adiabatic. (see Meakin \& Arnett (2006a), Meakin \& Arnett (2006b)).

Fig. 12 shows this decrease in wave energy. Using velocity scaling from our simulations, we estimate that roughly 0.05 to 0.1 of a solar luminosity may be moving inward as are these waves, and being deposited as shown. This would exactly mimic an enhanced opacity. However, the details depend upon the spectrum of waves, and their penetration is sensitive to their frequency and wavenumber. The standard solar model may need revision in this respect.

In addition, the waves will enhance gravitational settling (diffusion), which tends to help remove the helioseismological discrepancy. Both these effects are largest in the region of the sun where the discrepancy is largest.

\section{Conclusions}

3D simulation of stellar convection are beginning to provide interesting results for stellar astronomy. We will be able to predict the mixing length for near adiabatic convection, and the entrainment rate for turbulent convection. We have shown (Meakin \& Arnett (2006b)) that anelastic and our compressible codes give consistent results for oxygen burning shells, given the same computational domains. The anelastic codes are limited only by the flow velocity, not the sound speed, and so can take larger timesteps. If they can be generalize to use multiple fluids (ashes and fuel, eg.), then we can use larger domains, and perhaps include rotation and MHD.

\section{Acknowledgements}

This work was begun with the notion of collaborating with John Bahcall on extending the standard solar model; we hope John would have liked it. We thank Evry Schatzman, Ed Spiegel, Jean-Paul Zahn, and Bill Press for showing the way, Steward Observatory for our use of the Beowulf computer cluster, and the organizers for the invitation to 

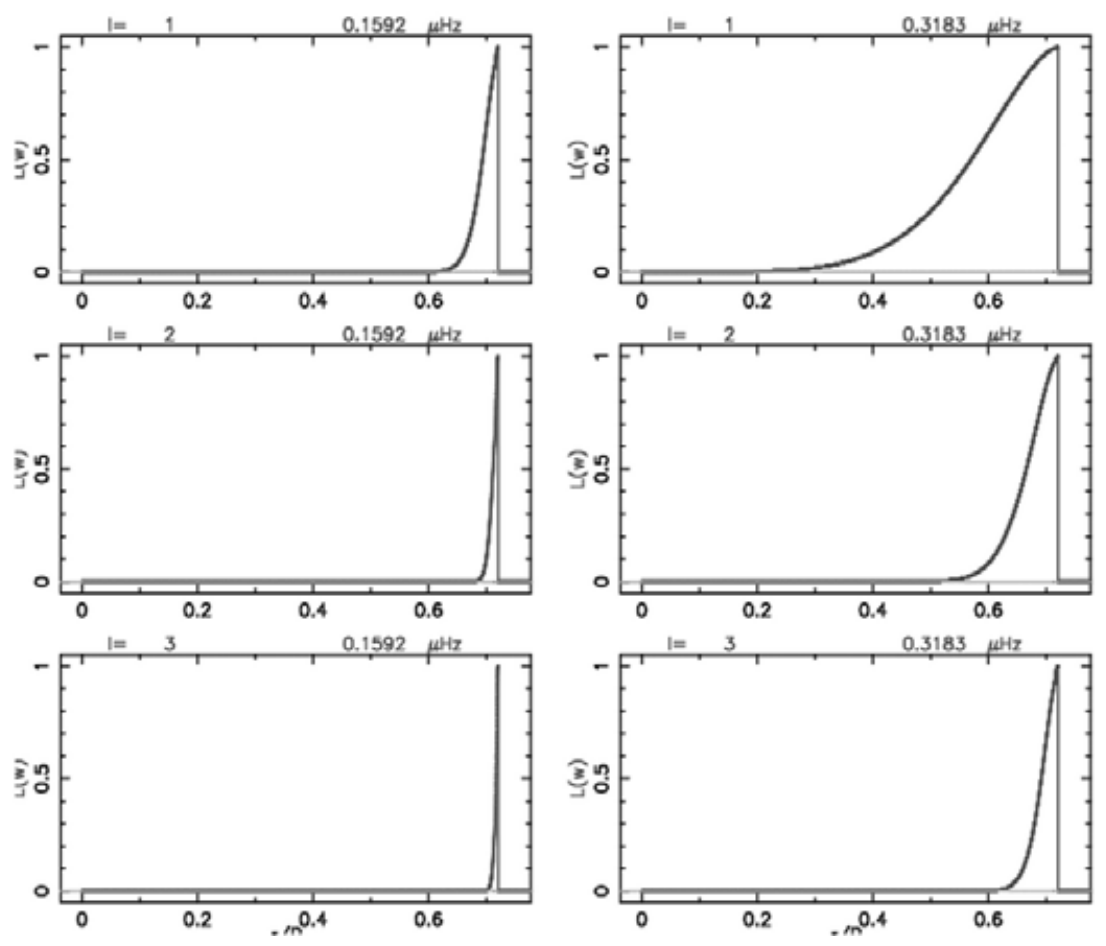

Figure 12. Amplitudes for waves generated at the lower boundary of the solar convection zone. The left column has a frequence equal to that of convection at the bottom of the convection zone $(0.016 \mu \mathrm{Hz})$; the right column, twice that. The top row has angular eigenvalue $\ell=1$, the middle and bottom rows are 2 and 3, respectively. (see Meakin \& Arnett (2006a), Meakin \& Arnett (2006b)).

participate. We would like to acknowledge support under under a subcontract from the FLASH Center at the University of Chicago.

\section{References}

Arnett, D. 1996, Supernovae and Nucleosynthesis, Princeton University Press, Princeton Kippenhahn, R. \& Weigert, A. 1990, Stellar Structure and Evolution., Springer-Verlag, Berlin, p. 41

Meakin, C. \& Arnett, D. 2006a, ApJ 637, 53

Meakin, C. \& Arnett, D. 2006b, ApJ, submitted

Meakin, C. \& Arnett, D. 2006c, ApJ, in preparation

Press, W.H. 1981, ApJ 245, 286

Unno, W., Osaki, Y., Ando, H., Saio, H. \& Shibahasi, H. 1989, Nonradial Oscillations of Stars, Tokyo: University of Tokyo Press, 2nd. Ed.

\section{Discussion}

RoxBurgh: Could you tell me about the time scales - turnover, thermal, and chemical?, and could you say what boundary conditions your used, and are the results robust against changes in the boundary conditions and size of the domain?

ARnETT: (1) The turnover times are the order of 100 seconds for the oxygen burning shell, the thermal time scales are comparable, and the chemical (I would say nuclear) timescale is about 10,000 seconds. 
(2) The boundary conditions are (top) stress-free, (bottom) reflecting, and (sides) are periodic. We determined our domains by trial and error to compromise between the smallest domain size and the smallest error. The results are robust against changes in domain size. Other choices of boundary condition tend toward ours as resolution is increased. However, there is an interesting question concerning standing waves or traveling waves which is nonlocal in nature; it can be messy.

V. M. Canuto: Which criterium do you use to determine the extent of overshooting?

ARnETT: We solve a physically "complete" problem, so the overshoot just happens, we do not impose it. The way we identify it is through our composition gradients, as the velocity contains both wave and mixing components, and is thus harder to disentangle.

J. Christensen-Dalsgaard: Comment: to compensate for the new solar abundances we need to increase the effective opacity at least down to $0.5 R_{\odot}$.

Question: When you talk about 'diffusion', I assume that you think about settling. What is the physical origin of the increase in settling due to waves?

ARnETT: Reply to comment: Yes, we find the same thing. Unfortunately we cannot as yet rule out a wave spectrum with some components getting that deep.

Gravitational settling is enhanced in second order, as Press (1981) showed. Think of the waves as sifting the atoms, with the heavier settling slightly faster. I used a roughly analogous device as a boy to sort grass seed..

J.-P. ZAHN: What thermal diffusivity are you using in your 3D simulations? In other words, what is the value of your Péclet number?

ARNETT: I have thought about this, but am not satisfied with my answer. We use OPAL opacity with radiative diffusion to determine thermal diffusivity, but in neutrino cooling stages, it is too small to be numerically relevant.. The Péclet number is the ratio of advection to diffusion. Where there is fluid motion, it dominates the flow, so I think the answer is $P_{e}>>1$. However, with neutrino cooling stages we do not even need to define the heat diffusion because it is the neutrino cooling and nuclear heating which dominate, and it is not yet clear to me what the corresponding physically useful parameter is. I hope that helps.

KWING L. ChAn: In the MLT comparison, what is the total depth of the convection zone in pressure scale heights? and, What is $F_{\text {convection }} / F_{\text {total }}$ ? These two questioon are raised because the "effective mixing length" may decrease with lower depth and lower efficiency.

ARneTt: The total depth of the oxygen shell, measured in pressure scale heights, is about 1.6. The ratio of convective flux to total flux is near unity in oxygen burning convective regions (radiative fluxes are orders of magnitude lower than convective).

However, in stellar evolution, the effective mixing length usually is adjusted to get the entropy jump right in the solar envelope (as you well know). I think that we should distinguish between the ratio of "kinetic energy per unit mass" to "thermal energy in the adiabatic excess", which we have determined for nearly adiabatic convection, and the parameter we use to fit the solar entropy jump. They need not be the same. 